Check for updates

Cite this: RSC Adv., 2017, 7, 35938

Received 1st May 2017

Accepted 4th July 2017

DOI: $10.1039 / \mathrm{c} 7 \mathrm{ra04866a}$

rsc.li/rsc-advances

\section{Preparation and therapeutic evolution of Ficus benjamina solid lipid nanoparticles against alcohol abuse/antabuse induced hepatotoxicity and cardio-renal injury}

\author{
Arun K. Sharma, $\mathbb{D} \dagger^{\star a}{ }^{\star a}$ Ashish Kumar, ${ }^{\mathrm{b}}$ Shantanu Kumar, ${ }^{\mathrm{b}}$ Siddhartha Mukherjee, ${ }^{\mathrm{b}}$ \\ Dheeraj Nagpal, ${ }^{\mathrm{b}}$ Upendra Nagaich ${ }^{\mathrm{b}}$ and Satyendra K. Rajputł ${ }^{\mathrm{a}}$
}

Chronic alcoholism destroys the propensity for hepatic detoxification of toxic substances, which further promotes the rapid and intense accumulation of aldehydes in the liver. Subsequently, chronic alcoholism exerts fatal liver and cardio-renal injury caused by fat deposition, dyslipidemia, inflammation and oxidative stress. The consequence becomes more perilous in disulfiram (potent aldehydes dehydrogenase inhibitor)-allied alcoholism, which further increases hepatic aldehyde accumulation by inhibiting aldehyde dehydrogenase. Ficus benjamina ( $F$. benjamina) is rich in phenolic and total flavonoid content and demonstrates effective antioxidant, anti-inflammatory, antinociceptive, antipyretic, hypotensive, anti-dysentery and antimicrobial properties. Numerous formulations of Ficus benjamina solid lipid nanoparticles (FBSLNP) were prepared and characterized using several physicochemical and drug stability parameters including entrapment efficiency, particle size, zeta potential, and TEM and in vitro drug release studies. In vivo evaluation of FBSLNP was carried out against hepatic and cardio-renal injury induced by concurrent administration of alcohol and disulfiram. Imperative recoveries in studies involving biological parameters including SGPT/SGOT, alkaline phosphatase level, lipid profile, serum protein and oxidative stress, and histological photomicrography revealed the hepatoprotective action of FBSLNP. Reduced levels of accumulated aldehydes in liver tissue were observed via gas chromatography, which confirmed the detoxifying nature of FBSLNP. Moreover, the restoration of aberrant cardio-renal biomarkers (CKMB, LDH, cTnl and serum creatinine, blood urea nitrogen, and microproteinuria) and histological consequences significantly reveal the cardio-renal protective potential of FBSLNP against alcohol abuse/antabuse-associated injury. Considering the results, it may conclude that the significant protection by FBSLNP is based on its anti-oxidant, anti-inflammatory and detoxification potential with regard to accumulated hepatic aldehydes.

\section{Introduction}

Alcohol is a psychoactive drug that can result in dependence. A WHO report (2014) disclosed that alcoholism has a high mortality rate of about 3.3 million worldwide and creates a huge global burden on health. ${ }^{\mathbf{1 , 2}}$ More specifically, chronic alcoholism provokes life-threatening liver diseases including steatosis, fibrosis, hepatocellular carcinoma, and cirrhosis. In addition, chronic alcoholism has been found to be a primary cause of cardiovascular and renal injury.,4 Conversely, the human body has the ability to metabolize the consumed alcohol

${ }^{a}$ Cardiovascular Pharmacology Division, Department of Pharmacology, Amity University, Noida, Uttar Pradesh 201303, India. E-mail: arunpharma2013@gmail. com; Tel: +91 1204392498 ext. 5557

${ }^{b}$ Department of Nanomedicine and Pharmaceutics, Amity University, Noida, Uttar Pradesh 201303, India

$\dagger$ Both authors have equal contribution. into aldehydes in the presence of alcohol dehydrogenase enzyme. The aldehydes produced trigger the impairment of proteins, enzymes, as well as DNA, and thus encourage mutagenesis and fibrogenesis in the liver. Conventionally, aldehydes can be metabolized to carboxylic acids by the aldehyde dehydrogenase enzyme. These acids are eliminated from the body, but with a slow metabolism rate. ${ }^{1}$ Slow metabolism of aldehydes leads to accumulation of aldehydes in hepatic tissue. This accumulation significantly rises in the presence of disulfiram, which is a potent aldehyde dehydrogenase inhibitor. Accumulated aldehydes in hepatic tissue considerably increase the prevalence of hepatitis and liver necrosis. Thus, the consequence of liver damage becomes more severe in persons who try to abstain from the consumption of alcohol using antabuse (disulfiram)., ${ }^{5,6}$ This alcohol/aldehyde accumulation or delayed clearance due to enzymatic diminution by disulfiram causes double toxicity to the liver. 
Ficus benjamina (F. benjamina) is a flowering plant (family Moraceae) found in India, Malaysia and Australia. This plant is rich in phenolic (chlorogenic, $p$-coumaric, ferulic and syringic acids), and flavonoids compounds which exhibits effective antioxidant, anti-inflammatory, antinociceptive, antipyretic, hypotensive, anti-dysentery and antimicrobial properties. ${ }^{7,8}$ Moreover, $F$. benjamina is also a rich source of alkaloids, triterpenes, and ascorbic acid. ${ }^{9}$ Indeed, with its various constituents having potent therapeutic applications, $F$. benjamina may play a significant role in hepatoprotection, renal protection and cardiovascular protection. ${ }^{\mathbf{1 0}}$ However, high dose of $F$. benjamina has been reported to cause sub-acute toxicity in liver after administration for prolonged duration. ${ }^{11}$

Nano-formulations with particle sizes more than $60 \mathrm{~nm}$ cannot be uptaken by the reticuloendothelial (RES) system when administered by the intravenous route. Thus, conventional nanocarriers are promptly cleared from the circulatory system following intravenous injection ( $90 \%)$ by RES organs, predominantly by fixed macrophages present in the liver. Solid lipid nanoparticles (SLNP) are an alternative colloidal carrier material generated by submicron-sized lipid emulsions. ${ }^{12}$ They have unique properties such as smaller sizes, larger surface areas, high drug loading capacity and high phase interaction ability at interfaces, which can potentially improve the performance of therapeutic formulations and significantly reduce the effective dose concentration. ${ }^{\mathbf{1 2}}$ Moreover, being lipophilic in nature, they increase the possibility of RES uptake; hence, an improved localization of a given drug at the target site can be achieved by SLNP. Thus, SLNP of $F$. benjamina (even in low dose) is enough to produce potential effect that can significantly overcome subacute toxicity. The present study is focused on the preparation, characterization and in vitro/in vivo evaluation of SLNP of $F$. benjamina against hepatic and cardio-renal toxicity induced by the individual or co-administration of alcohol abuse and antabuse (disulfiram).

\section{Material and methods}

\subsection{Collection and identification of $F$. benjamina}

The leaves of $F$. benjamina plant were collected from the medicinal herb garden, Amity University, Noida, India and were authenticated by Dr M. P. Sharma, Department of Botany, Jamia Hamdard University, Delhi, by comparing sample leaves against a voucher specimen.

\subsection{Preparation of $\boldsymbol{F}$. benjamina extract}

Fresh leaves of $F$. benjamina were collected and washed with cold water and then distilled water. The leaves were cut into small pieces and shade dried at room temperature. Furthermore, $80 \mathrm{~g}$ of the dried leaves were crushed to form a fine powdered and were extracted using ethanol $(2.850 \mathrm{~L})$ at $79{ }^{\circ} \mathrm{C}$ for $24 \mathrm{~h}$ and filtered through Whatman filter paper (no. 1).

\subsection{Preformulation screening of $F$. benjamina extract}

Preformulation evaluation including melting point (MP, measured using an oil immersion melting point apparatus), solubility (SL, studied by using different solutions to find out the suitable carrier for the formulation), partition co-efficient (PC) and FTIR (for determination of the physicochemical properties of the extract, including drug and excipient interaction study) were evaluated for $F$. benjamina extract.

(1) Predetermined samples of $F$. benjamina $(10 \mathrm{mg})$ were each dissolved in $10 \mathrm{~mL}$ of different solvents (water, methanol, ethanol, acetone, chloroform, and benzene; $\mathrm{pH}$ adjusted to 7.4 with phosphate buffered saline (PBS), and $0.1 \mathrm{M} \mathrm{HCl}$ ), to obtain samples of $1 \mathrm{mg} \mathrm{mL} \mathrm{mL}^{-1}$ concentration. Serial dilutions of these solutions of $10,20,30,40,50,60,70,80,90$, and $100 \%$ were developed, and calibration graphs were generated.

(2) For our solubility study, excess samples were dissolved in the solvents of different polarities until saturated solutions were achieved. The samples were allowed to equilibrate over $24 \mathrm{~h}$. Then, $1 \mathrm{~mL}$ of each sample was taken and diluted into $10 \mathrm{~mL}$ of solvent, and the amount of drug dissolved in the solvent was calculated by interpolation of the calibration graph.

The partition co-efficient (PC) of the sample was determined by dissolving $10 \mathrm{mg}$ in $40 \mathrm{~mL}$ of a $1: 1$ solution of water : $n$ octanol. The sample was continuously stirred using a magnetic stirrer for $2 \mathrm{~h}$. Furthermore, $10 \mathrm{~mL}$ of the aqueous phase was withdrawn and the amount of sample dissolved in the aqueous phase was determined by UV-vis spectroscopy and interpolation of the calibration graph.

Moreover, FTIR spectroscopy was carried out using the $\mathrm{KBr}$ press pellet technique for determining the physicochemical properties of the extract including drug:excipient interaction study. The $F$. benjamina extract and sample of excipients were analyzed via FTIR spectroscopy and compared with the FTIR spectra of the drug:excipient combination (the drug and excipients were stored for 3 months at ambient temperature and humidity conditions).

\subsection{Preparation of $F$. benjamina solid lipid nanoparticle (FBSLNP)}

FBSLNP were prepared by hot-homogenization, followed by ultra-sonication. The ingredients used for the formulation included stearic acid (lipid base), soy-lecithin (surfactant) and de-ionized water (dispersion medium). Briefly, the embedded lipid (lipid with entrapped $F$. benjamina) was melted by heating to $10{ }^{\circ} \mathrm{C}$ (above melting point of lipid). Conversely, an aqueous phase was prepared by dissolving the soy-lecithin (stabilizer) in distilled water at $10^{\circ} \mathrm{C}$. The hot aqueous phase was mixed with the oil phase and homogenized at $25000 \mathrm{rpm}$ for 10 minutes. This emulsion was sonicated using an ultrasonicator (Life Care Equipments Pvt. Ltd) for 30 minutes and finally the product was allowed to cool at room temperature and FBSLNP suspension was obtained. Lyophilization of the SLNP slurry formulation was carried by lyophilizer (MRCLAB) for $24 \mathrm{~h}$ at a temperature of $-49{ }^{\circ} \mathrm{C}$, and a white powder was collected and stored in desiccators for further evaluation.

\subsection{Characterization/evaluation of solid lipid nanoparticles (entrapment efficiency/particle size/zeta potential)}

The entrapment efficiency of various formulations of FBSLNP dispersion was determined via a centrifugation method. In 
brief, SLNP dispersion having $0.5 \mathrm{~mL}$ of $F$. benjamina was centrifuged at $9000 \mathrm{rpm}$ for 30 minutes, and the supernatant was collected. The supernatant was filtered through a Whatman filter paper (grade 1), and the free drug concentration was measured using a UV-spectrometer at $204 \mathrm{~nm}$ to determine the entrapment efficiency. Morphological evaluation and measurement of particle size of various formulations were carried out via dynamic light scattering (DLS) using a Malvern Zetasizer instrument (Malvern Instruments, Malvern, UK). The zeta potential was used for determination of the surface charge of the nanoparticles. The zeta potential of various formulations of FBSLNS were determined by the measurement of electrophoretic light scattering (ELS) in the Zetasizer instrument. The samples were prepared by dilution with ultra-pure water and analyzed at $25{ }^{\circ} \mathrm{C}$.

\subsection{In vitro drug release studies of SLNs}

2.6.1 Determination of $\lambda_{\max }$. For the determination of $\lambda_{\max }$, $10 \mathrm{mg}$ of the $F$. benjamina extract was dissolved in freshly prepared PBS. The solution was scanned using UV-vis spectrophotometry for determination of $\lambda_{\max }$. Serial dilutions of the solution were prepared, and the calibration graph was plotted.

In vitro release study was performed on freshly prepared FBSLNP. Furthermore, $2 \mathrm{~mL}$ of dispersion was transferred to a dialysis bag, introduced into a beaker containing $900 \mathrm{~mL}$ of a phosphate buffer solution at $37 \pm 1{ }^{\circ} \mathrm{C}$ and stirred at $100 \mathrm{rpm}$. At predetermined time intervals $(0,1,2,3,4,8,12,16,20,24 \mathrm{~h})$, $10 \mathrm{~mL}$ sample of the medium was withdrawn and replaced with the same amount of fresh medium. The percentage cumulative drug release (CDR) from the SLN was quantified by UV-vis spectrophotometry at a wavelength of $206 \mathrm{~nm}$. The concentration of FBSLNP in the test samples was calculated via interpolation of the calibration curve.

\subsection{Transmission electron microscopy (TEM)}

TEM was carried out for selected formulations to determine the internal properties of the prepared nanoparticles. The samples were prepared by diluting $1 \mathrm{~mL}$ of FBSLNP dispersion with 10 $\mathrm{mL}$ of $\mathrm{pH} 7.4$ phosphate buffer and were centrifuged at $4000 \mathrm{rpm}$ for 5 minutes. They were then sent to IIT Roorkee for further evaluations for particle size and morphological evaluations of the prepared FBSLNP.

\subsection{In vivo study}

FBSLNP was evaluated against alcohol and disulfiram-induced hepatic, cardiac and renal toxicity.

2.8.1 Induction and assessment of alcohol and disulfiraminduced toxicity (hepatic, cardiac and renal toxicity) in rats. Animals were routinely exposed to chronic alcoholism (ethanol, $16 \mathrm{~g}$ per kg per day, p.o) for 4 weeks, ${ }^{13}$ followed by administration of a potent aldehyde dehydrogenase inhibitor (disulfiram, $\mathbf{1 2 . 5} \mathrm{mg}$ per $\mathrm{kg}$ per day, p.o $)^{\mathbf{1 4}}$ to induced hepatic toxicity due to direct exposure of alcohol to the liver and deposition of aldehyde in hepatic cells. Deposition of aldehyde in hepatic cells was estimated by a headspace gas chromatography (GCHS) technique. This chronic exposure to alcohol and disulfiram caused significant renal and cardiovascular abnormality. Hepatic and cardio-renal toxicity was induced in alcoholic rats over a duration of 4 weeks, the extents of which were measured by estimating various biochemical and histological parameters.

2.8.2 Experimental protocol. The experimental protocol was designed by categorizing the animals into eight groups containing eight rats each. Group 1 (normal control group) animals received blank SLNP, (50 mg per kg per day, p.o (having no drug molecule)) throughout the entire study duration, and group 2 (alcohol treated group) received alcohol (16 g per kg per day, p.o) for four weeks. Similarly, group 3 (disulfiram treated group) received disulfiram (12.5 $\mathrm{mg}$ per $\mathrm{kg}$ per day, p.o) for four weeks. For group 4, (disulfiram + alcohol treated group) alcohol (16 g per kg per day, p.o) was given to disulfiram (12.5 mg per kg per day, p.o) pretreated rats for four weeks. Group 5 (FBSLNP per se group) received FBSLNP (50 mg per kg per day, p.o) for four weeks. Group 6 (FBSLNP + alcohol treated group) received FBSLNP (50 mg per kg per day, p.o) for four weeks after $2 \mathrm{~h}$ of administration of alcohol. Group 7 (FBSLNP + disulfiram treated group) received FBSLNP (50 mg per $\mathrm{kg}$ per day, p.o) for four weeks after $2 \mathrm{~h}$ of administration of disulfiram. For group 8 (FBSLNP + disulfiram + alcohol treated group), FBSLNP (50 mg per $\mathrm{kg}$ per day, p.o) was administered for four weeks after $2 \mathrm{~h}$ of alcohol and disulfiram treatment, as mentioned in group 4 .

The animal protocol for the present study was approved by IAEC (IAEC/1107/ADH/089) as per the norm of CPCSEA, New Delhi, India. Adult Wistar albino rats (250-300 $\mathrm{g}$ of either sex) were obtained from the institutional animal house and acclimatized at normal laboratory conditions $\left(25 \pm 1{ }^{\circ} \mathrm{C}\right.$ temperature; $45-55 \%$ relative humidity and normal day/night cycle) for 15 days prior to the start of the experiment. Standard rat food and water was supplied to the animals ad libitum.

2.8.3 Assessment of biochemical parameters. Blood was collected (from the tail vein) from all the animal groups and was allowed to clot at room temperature; serum was separated by centrifugation at $2500 \mathrm{rpm}$ for $15 \mathrm{~min}$. The serum was used for estimation of biochemical parameters to determine the functional state of the liver. Serum glutamic oxaloacetic transaminase (SGOT), serum glutamic pyruvic transaminase (SGPT) and alkaline phosphatase (ALP) were measured using commercially available kits (Sigma-Aldrich, US). ${ }^{15}$ All other biochemical factors including lipid profile (total cholesterol, triglyceride and high-density lipoprotein) and serum protein were assessed using commercially available kits (Sigma-Aldrich, US). Protein estimation was carried out by measuring albumin and total protein. Hepatic thiobarbituric acid reactive species (TBARS) and reduced glutathione (GSH) were quantified for oxidative stress determination. Creatinine kinase MB (CKMB), lactate dehydrogenase $(\mathrm{LDH})$, cardiac troponin I (cTnI) and serum creatinine, blood urea nitrogen (BUN), and microproteinuria were measured for estimating the cardiovascular and renal damage, respectively, using commercially available kits (Sigma-Aldrich, US). ${ }^{16}$

2.8.4 Quantification of alcohol and acetaldehyde in the liver by GC-HS. An Elite-WAX $(30 \mathrm{~m} \times 0.32 \mathrm{~mm} \times 0.5 \mu \mathrm{m})$ capillary column with an auto sampler 7890A GC-HS system (Gas Chromatograph with TurboMatrix HS-40 headspace 
analyzer) was used to detect the accumulated hepatic alcohol and acetaldehyde. The homogenized liver tissue sample was weighed $(1 \mathrm{~g})$ and diluted $\times 10$ with phosphate buffer. Furthermore, $100 \mathrm{mg}$ of supernatant was transferred to head space vials. The screw cap was closed after adding $2 \mathrm{~mL}$ of water. Samples were prepared in duplicate fashion. Quantification was carried out using a flame ionization detector $\left(200{ }^{\circ} \mathrm{C}\right)$ with nitrogen as the carrier gas (mobile phase; flow: $0.5 \mathrm{~mL} \mathrm{~min}^{-1}$ ).

2.8.5 Histological examination. After termination of the experimental protocol, animals from each group were sacrificed. The vital organs (liver, heart and kidney) were dissected out and were stored in $10 \%$ neutral formalin solution embedded in paraffin wax. The sections obtained after staining with hematoxylin and eosin for 20-40 minutes were observed under an inverted microscope (Cosmo Laboratory Equipment).

2.8.6 Statistical analysis. Statistical analysis was carried out using one-way ANOVA, followed by Tukey's multiple comparison tests (Sigma Plot version 11.0, from Systat Software, Inc., San Jose California USA). All values were expressed in mean \pm SD. ${ }^{* * *} P<0.001, * * P<0.01, v s$. normal control, @@@ $P<0.001$, @@ $P<0.01$, vs. alcohol treated, ${ }^{\# \# \#} P<0.001,{ }^{\# \#} P<0.01$, vs. disulfiram treated, ${ }^{\$ \$ \$} P<0.001,{ }^{\$ \$} P<0.01$ vs. disulfiram + alcohol treated were consider as statistical significant data.

\section{Results}

\subsection{Evaluation of preformulation of $F$. benjamina extract}

The physicochemical properties of the extract including melting point (MP), solubility (SL), and partition co-efficient (PC) are given in Table 1. FTIR spectra of $F$. benjamina extract (Fig. 1A) and excipients samples (Fig. 1B) and their combination (Fig. 1C) stored for 3 months at room temperature and humid conditions $\left(25{ }^{\circ} \mathrm{C} \pm 2{ }^{\circ} \mathrm{C}, \mathrm{RH} 60 \% \pm 5 \%\right.$ ) were analyzed. The analyzed spectra of the stored combined sample of drug and excipients showed no new peaks compared to the $F$. benjamina extract FTIR spectra of each component, which indicates the absence of new products and confirms the product compatibility with no interactions with each other, as shown in Fig. 1A-C.

Table 1 Physicochemical parameters (colour, odour, melting point, solubility and partition coefficient) of $F$. benjamina extract

S. no. Physicochemical parameter Observation

$\begin{array}{lll}1 & \text { Morphologic description } & \begin{array}{l}\text { The extract was blackish-brown in } \\ \text { colour and had characteristic odour }\end{array} \\ 2 & \text { Melting point } & 127-130^{\circ} \mathrm{C} \\ 3 & \text { Solubility } & \text { (Concentration of extract, } \mathrm{mg} \mathrm{mL}^{-1} \text { ) } \\ \text { (I) Distilled water } & 7.02 \\ \text { (II) Methanol } & 15.13 \\ \text { (III) Ethanol } & 16.95 \\ \text { (IV) Acetone } & 10.34 \\ \text { (V) Chloroform } & 7.21 \\ \text { (VI) Benzene } & 3.13 \\ \text { (VII) Phosphate buffer } & 10.85 \\ & \text { (pH - 7.4) } & \\ \text { (VIII) HCl (0.1 N) } & 2.85 \\ 4 & \text { Partition coefficient } & 0.0943\end{array}$

\subsection{Preparation of FBSLNP}

Various formulations with different compositions of ingredients were developed, as shown in Table 2.

\subsection{Evaluation of solid lipid nanoparticles (entrapment efficiency/particle size/zeta potential)}

Entrapment efficiency plays a crucial role in determining the method of preparation and composition of a formulation. In order to obtain optimum entrapment efficiency, various compositions were evaluated. The entrapment efficiency of all compositions ranged from $68-93 \%$, whereas formulation $\mathrm{FE}$ had the highest entrapment efficiency, as shown in Fig. 2A. Morphological evaluation and particle size determination are essential parameters for confirming the production of nanosize particles. The individual nanoparticle size of FBSLNP from different formulations was measured by DLS and found to be in the range of 44 to $403 \mathrm{~nm}$ as depicted in Fig. 2A. Specifically, the FE formulation of FBSLNP was found to have the smallest particle size compared to the other formulations. In addition, the surface potential of nanoparticles is a crucial parameter for stability within biological systems which may lies in between $\pm 30 \mathrm{mV}$ charges for better stability and compatibility. The measured zeta potential ( $\zeta$-potential) of various formulations of FBSLNP are shown in Fig. 2A, which defines the negative surface charge ranging between -25.6 and $-39.4 \mathrm{mV}$. Again, FE formulation of FBSLNP showed a desired zeta potential of $-32.7 \mathrm{mV}$.

\subsection{In vitro drug release studies of FBSLNP}

An alkaline medium, PBS ( $\mathrm{pH}$ 7.5) was used as the diffusion medium to study the release patterns between different FBSLNS formulations. The maximum percentage in cumulative drug release (\% CDR) for all FBSLNP formulations was in the range of $76 \%$ to $89 \%$. In the prepared SLNP, the lecithin : stearic acid ratio slightly influenced the release rate of SLNP because lecithin and stearic acid ratio might change the polymorphic state of lipid constitutes, inducing a difference in catalyzes solubility in lipid matrix, and change drug incorporation. The highest cumulative release was found in the FE formulation during a $12 \mathrm{~h}$ time period, as shown in Fig. 2B.

\subsection{Formulation selection for in vivo study}

Selection of more appropriate formulations of FBSLNP for further in vivo analysis was carried on the basis of entrapment efficiency, particle size and zeta potential, which are the prime factors for stability and therapeutic efficacy of nanoformulations. Formulation $\mathrm{FE}$ exhibited more favourable measurements for these crucial parameters. Moreover, the in vitro drug release pattern further marks the same formulation on the basis of \% CDR, and hence was selected for further in vivo evaluation.

\subsection{TEM analysis of selected formulation of FBSLNP (FE)}

TEM is high-definition microscopy based on a focused beam of electrons and provides various signals as per the sample's 


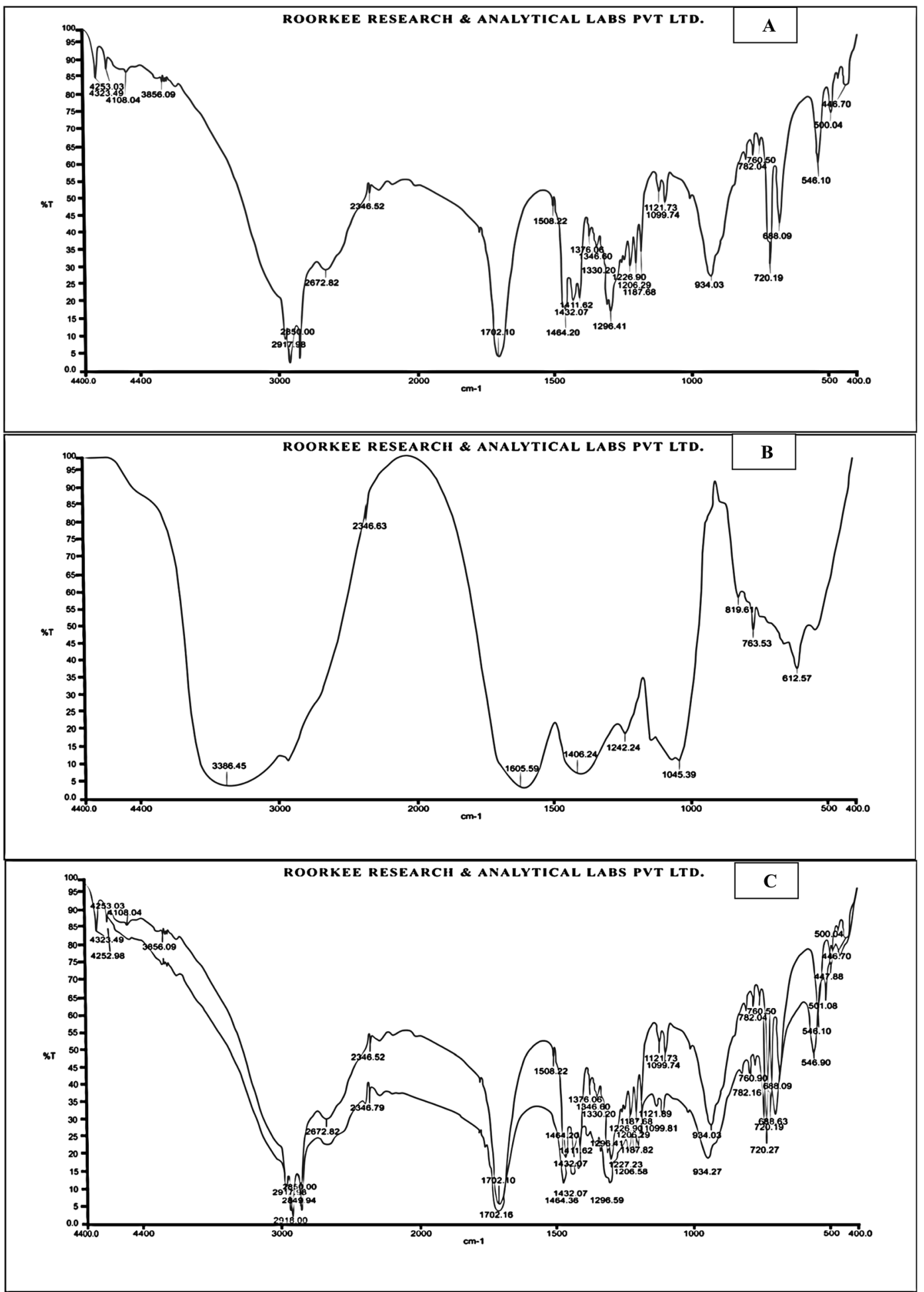

Fig. 1 FTIR spectra including F. benjamina extract represents in (A) and excipients samples represents in (B) and combination of both represents in (C) stored for 3 months at ambient temperature and humidity.

surface topography and composition. The TEM image (Fig. 2C) of a selected formulation (FE) of FBSLNP showed that all particles were roughly spherical in shape, with the size ranging from 20 to $50 \mathrm{~nm}$, due to the non-polar nature of stearic acid and possessed a more stable morphology.

\subsection{In vivo study}

3.7.1 Assessment of SGOT/SGPT and ALP. Assessment of SGOT/SGPT and ALP level was found to be increased in the alcohol and disulfiram treated animals either individually or in 
Table 2 Formulation composition of $F$. benjamina leaf extract loaded solid lipid nanoparticles

\begin{tabular}{|c|c|c|c|c|c|}
\hline \multirow[b]{2}{*}{ S. no. } & \multirow[b]{2}{*}{$\begin{array}{l}\text { Formulation } \\
\text { code }\end{array}$} & \multicolumn{4}{|c|}{ Ingredients } \\
\hline & & $\begin{array}{l}\text { Drug } \\
(\mathrm{mg})\end{array}$ & $\begin{array}{l}\text { Stearic } \\
\text { acid }(\mathrm{g})\end{array}$ & $\begin{array}{l}\text { Soy lecithin } \\
\text { (g) }\end{array}$ & $\begin{array}{l}\text { Distilled } \\
\text { water }(\mathrm{mL})\end{array}$ \\
\hline 1 & FA & 80 & 2.5 & 0.1 & 100 \\
\hline 2 & FB & 80 & 5 & 0.1 & 100 \\
\hline 3 & FC & 80 & 2.5 & 0.25 & 100 \\
\hline 4 & FD & 80 & 5 & 0.25 & 100 \\
\hline 5 & $\mathrm{FE}$ & 80 & 2.5 & 0.5 & 100 \\
\hline 6 & $\mathrm{FF}$ & 80 & 5 & 0.5 & 100 \\
\hline 7 & FG & 80 & 2.5 & 1.00 & 100 \\
\hline 8 & $\mathrm{FH}$ & 80 & 5 & 1.00 & 100 \\
\hline
\end{tabular}

combination, as compared to the normal group. However, a significant reduction was observed in the elevated levels of SGOT/SGPT and ALP in the FBSLNP treated group (FBSLNP + alcohol $v s$. alcohol treated, FBSLNP + disulfiram $v s$. disulfiram treated and FBSLNP + alcohol + disulfiram vs. alcohol + disulfiram treated animals). Moreover, there was no significant difference in the SGOT/SGPT and ALP levels with FBSLNP per se group animals compared to the control, as shown in Fig. 3A, B and $\mathrm{C}$ respectively.

3.7.2 Assessment of albumin and total protein. Levels of albumin and total protein were found to be significantly reduced in the alcohol and disulfiram treated animals either alone or in combination. Conversely, the reduced levels of albumin and total protein were extensively increased by FBSLNP treatment against alcohol and disulfiram treated animals (FBSLNP + alcohol vs. alcohol treated, FBSLNP + disulfiram vs. disulfiram treated and FBSLNP + alcohol + disulfiram $v s$. alcohol + disulfiram treated animals), as shown in Fig. 3D and E respectively.

3.7.3 Assessment of oxidative stress. A substantial decrease in the level of hepatic GSH and increase in the level of hepatic TBARS were found in alcohol and disulfiram treated animals, either alone or in combination, as compared to the control group, which strongly proves the increased level of hepatic reactive oxygen species (ground mediators of several pitfall signaling). FBSLNP per se treatment did not affect any oxidative marker. However, significant reduction in the malondialdehyde (MDA) level and substantial increase in the GSH level was observed for the FBSLNP treatment, which reveal the antioxidant potential of $F$. benjamina (Fig. 3F and G respectively).

3.7.4 Assessment of alcohol and aldehyde in liver cell. The concentration of accumulated aldehyde in the liver was measured in part per million (ppm) by analyzing defined tissue samples using a 7890A GC system containing an Elite-WAX (30 $\mathrm{m} \times 0.32 \mathrm{~mm} \times 0.5 \mu \mathrm{m}$ ) capillary column with an auto sampler. GC-HS showed the significant presence of accumulated acetaldehyde in the alcohol and disulfiram (in combination) treated animals compared to normal control animals, which clearly revealed the factor responsible for liver damage in the alcohol and disulfiram treated animals. Administration of FBSLNP significantly reduced the deposition of acetaldehyde in the alcohol and disulfiram treated animals either alone or in combination. This reduction in deposited acetaldehyde from the liver reveals the detoxifying property of FBSLNP, as shown in Fig. $3 \mathrm{H}$ and I respectively. The concentration of alcohol and acetaldehyde was calculated using the formula:
Alcohol or acetaldehyde $(\mathrm{ppm})=$
(area of sample/average area of standard)
$\times$ (dilution of standard/dilution of sample)
$\times$ (purity of standard) $\times 10000$

3.7.5 Assessment of serum lipid profile. The lipid profile in serum including total cholesterol, high-density lipoprotein, lowdensity lipoprotein and triglyceride (TC, HDL, LDL and TG) were found to be disrupted in the alcohol and disulfiram treated animals. TC, LDL and TG were significantly raised in the alcohol and disulfiram treated animals either alone or in combination. On the other hand, the HDL level was not significantly interrupted with the exposure of alcohol and disulfiram. Interestingly, treatment with FBSLNP reduced the increased level of TC, LDL and TG in the alcohol and disulfiram treated animals either alone or in combination (Table 3). Individually, FBSLNP did not show any interaction with the lipid profile.

3.7.6 Assessment of CKMB, LDH and cTnI. Substantially increased levels of CKMB, LDH and CTnI were observed in the alcohol and disulfiram treated animals either alone or in combination, compared to the control group of animals, which significantly indicates the presence of cardiac injury. Treatment with FBSLNP considerably decreased their levels compared to the alcohol and disulfiram treated animals (FBSLNP + alcohol vs. alcohol treated, FBSLNP + disulfiram vs. disulfiram treated and FBSLNP + alcohol + disulfiram vs. alcohol + disulfiram treated animals) either alone or in combination, as shown in Fig. 4A, B and C, respectively.

3.7.7 Assessment of serum creatinine, BUN, and microproteinuria. Serum creatinine, BUN and microproteinuria are critical biomarkers that indicate renal injury. The levels of serum creatinine and BUN were noted to be significantly raised in the alcohol and disulfiram treated animals either alone or in combination, compared to the control animal group that confirmed the existence of renal injury. Furthermore, the incidence of microproteinuria in the alcohol and disulfiram treated animals either alone or in combination, compared to the control group animals, proved renal damage. However, the treatment with FBSLNP prevented the significant elevation of serum creatinine and BUN and the occurrence of microproteinuria in the alcohol and disulfiram treated animals (FBSLNP + alcohol vs. alcohol treated, FBSLNP + disulfiram vs. disulfiram treated and FBSLNP + alcohol + disulfiram $v s$. alcohol + disulfiram treated animals) either alone or in combination, as shown in Fig. 4D, E and F respectively.

3.7.8 Histological assessment (liver, heart and kidney respectively). Histological examination of rat liver from the normal control did not show any structural alteration in hepatic lobule from the central vein and hepatic cords. Conversely, 

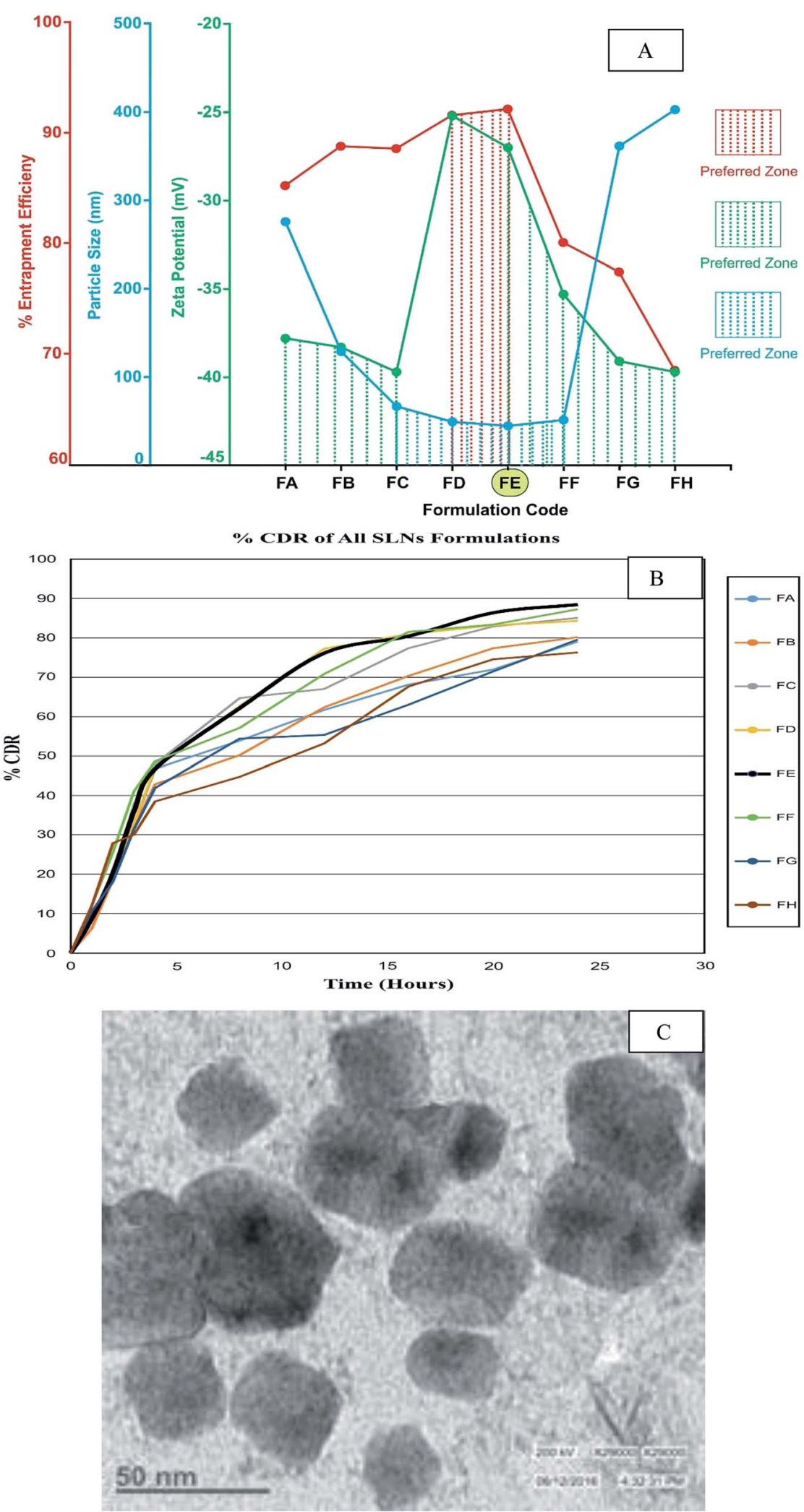

Fig. 2 Characterization of solid lipid nanoparticles of various formulations of FBSLNP including entrapment efficiency/particle size/zeta potential (A) and percentage in cumulative drug release (B) respectively. (C) represents the transmission electron microscopic analysis of a selected formulation (FE) of FBSLNP. 

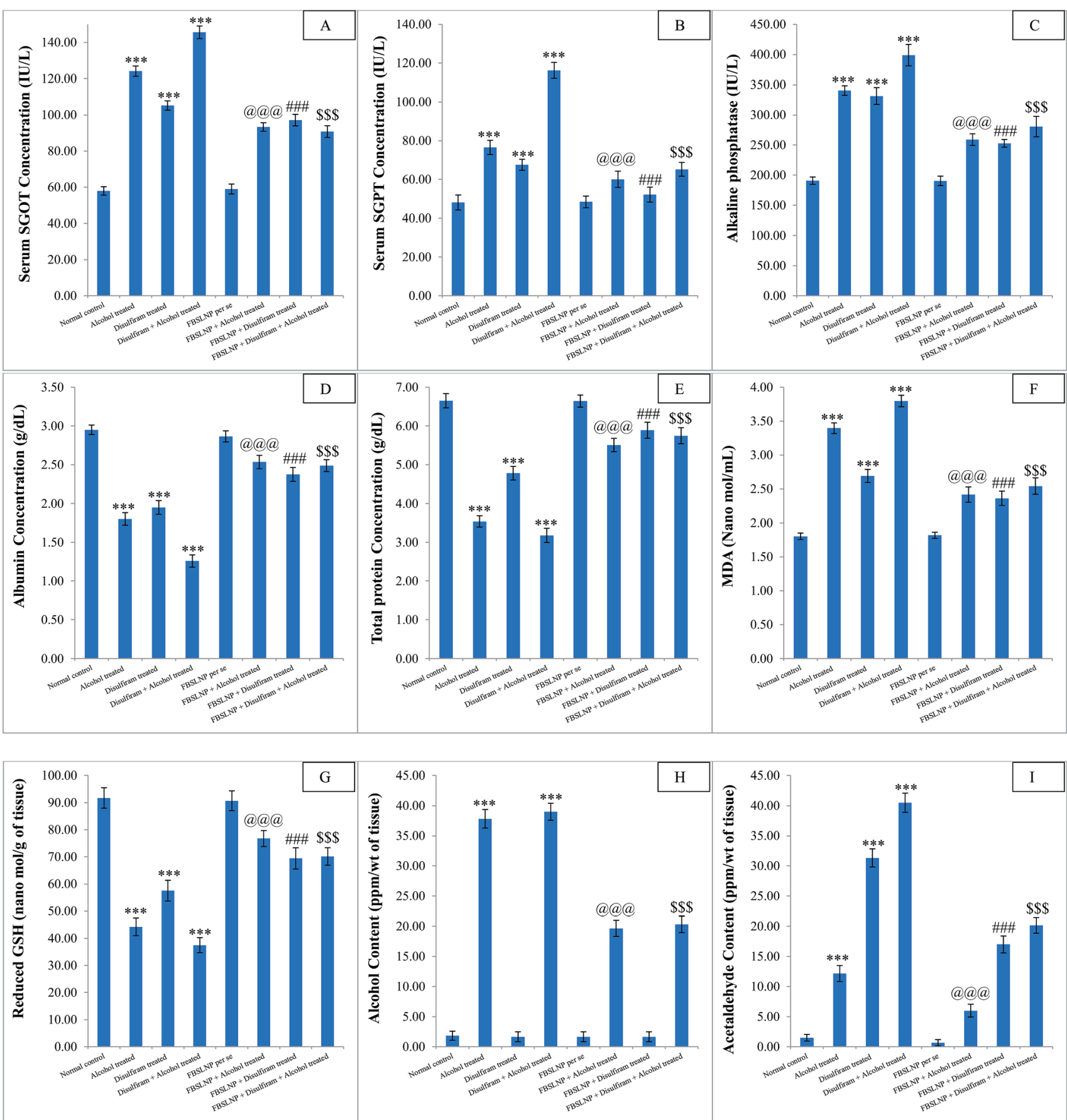

Fig. 3 Effect of FBSLNP against individual and combined treatments of alcohol and disulfiram in hepatocytes by measuring serum SGOT concentration in IU L $L^{-1}(A)$, SGPT concentration in $I U L^{-1}(B)$, alkaline phosphatase in $I U L^{-1}(C)$, serum albumin in $g \mathrm{dL}^{-1}(D)$, total protein in $g$ dL ${ }^{-1}$ (E), MDA in nano $\mathrm{mol} \mathrm{mL}^{-1}$ of hepatic tissue (F), reduced GSH in nano mol g-1 of hepatic tissue (G), alcohol content in ppm per wt of hepatic tissue $(\mathrm{H})$ and aldehyde content in ppm/wt of hepatic tissue (I). All data are expressed as mean $\pm \mathrm{SD}, * * * P<0.001, * * P<0.01, v s$. normal control, @®@ $P<0.001$, ${ }^{\circledR} P<0.01$, vs. alcohol treated, ${ }^{\# \#} P<0.001,{ }^{\# \#} P<0.01$, vs. disulfiram treated, $\$ \$ P<0.001$, vs. disulfiram + alcohol treated.

swelling of hepatocytes, Kupffer cell activation, karyomegaly, portal infiltration with inflammatory cells, dysplasia of the bile duct, hyperplasia and hyperactivation of the epithelial lining bile duct were observed in the alcohol and disulfiram treated animals individually and in combination. All these histological variations were significantly restored in the FBSLNP treated group against pretreated alcohol and disulfiram individually and in combination, as shown in Fig. 5(A-1 to A-8).

The histological section of the normal control rat heart ventricle portion showed no histological lesions. However, the histological examination of diseased group (alcohol and disulfiram either in alone or in combination) rats showed significant histological lesions including thrombus formation, myofibrillar 
Table 3 Serum concentrations of various lipid parameters, TC represents total cholesterol; HDL represents high density lipoprotein; LDL represents low density lipoprotein; TG represents triglycerides ${ }^{a}$

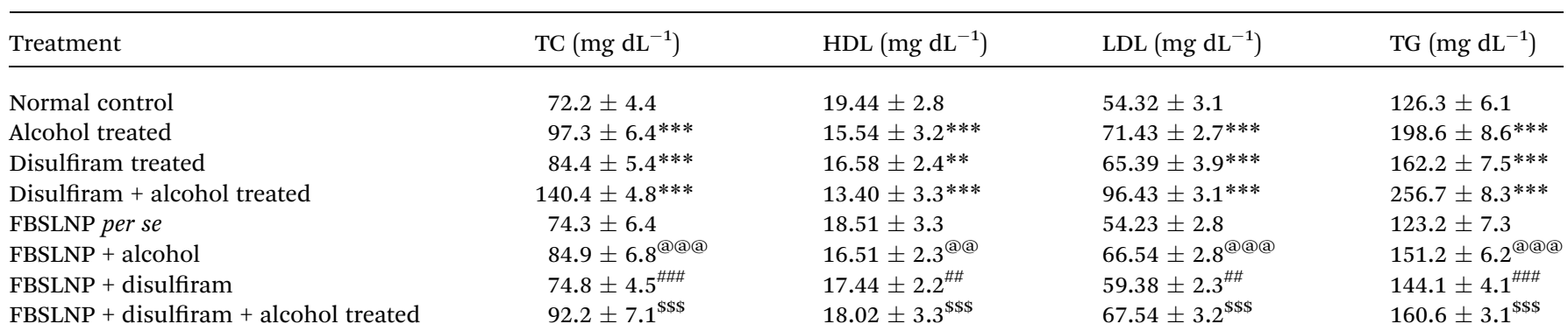

${ }^{a}$ All data are expressed as mean $\pm \mathrm{SD},{ }^{* * *} P<0.001,{ }^{* *} P<0.01, v s$. normal control, ${ }^{@ @ @ ~} P<0.001$, ${ }^{@} P<0.01, v s$. alcohol treated, ${ }^{\# \#} P<0.001,{ }^{\# \#} P<$ $0.01, v s$. disulfiram treated, ${ }^{\$ \$} P<0.001, v s$. disulfiram + alcohol treated.

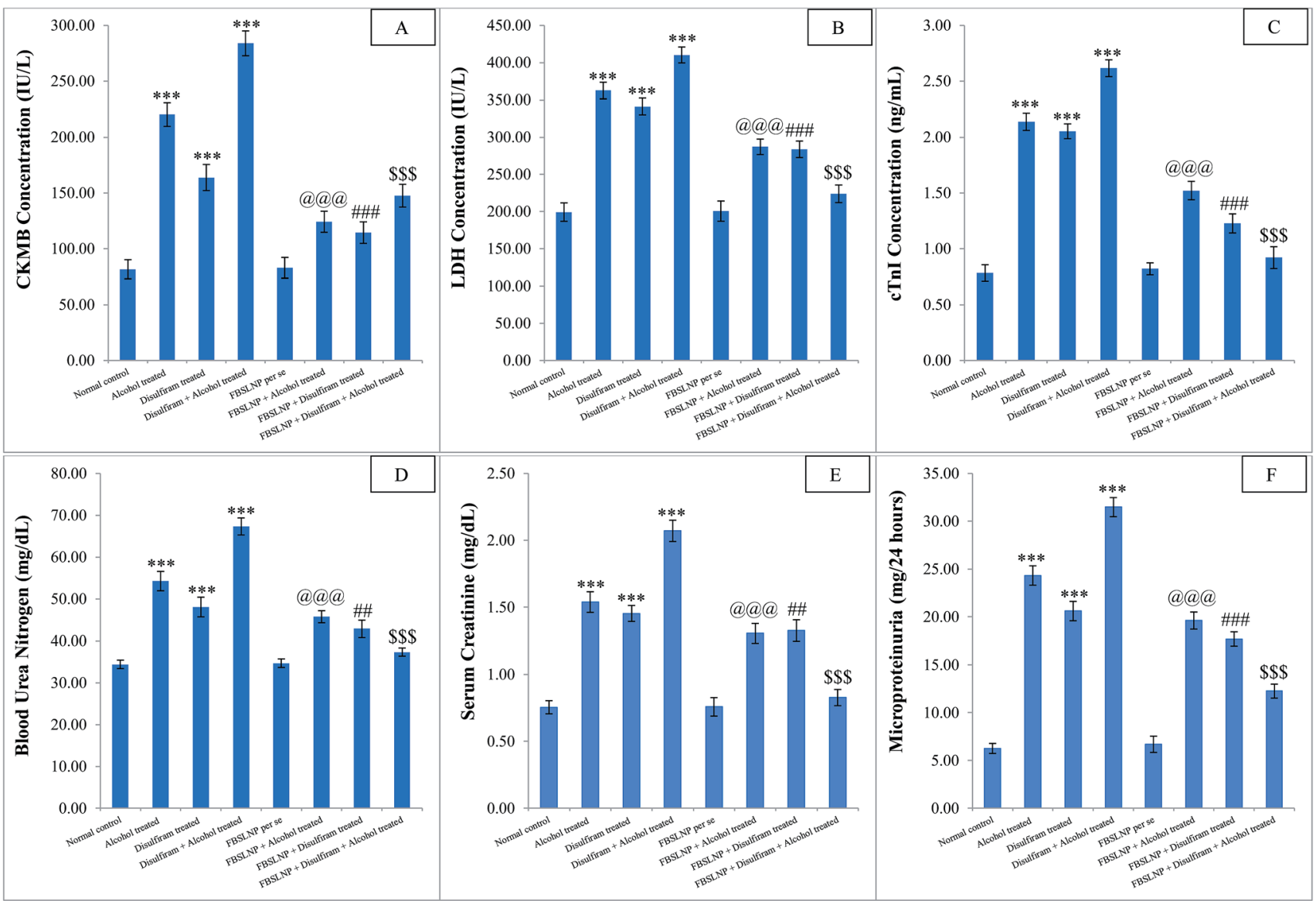

Fig. 4 The protective role of FBSLNP against individual and combined treatments of alcohol and disulfiram in cardio-renal tissue by measuring $\mathrm{CK}-\mathrm{MB}$ isoenzyme concentration in IU L $\mathrm{L}^{-1}(\mathrm{~A}), \mathrm{LDH}$ isoenzyme concentration in IU $\mathrm{L}^{-1}(\mathrm{~B}), \mathrm{cTnl}$ concentration in $\mathrm{ng} \mathrm{mL} \mathrm{L}^{-1}(\mathrm{C})$, blood urea nitrogen in $\mathrm{mg} \mathrm{dL}^{-1}(\mathrm{D})$, serum creatinine in $\mathrm{mg} \mathrm{dL}^{-1}(\mathrm{E})$ and microproteinuria in $\mathrm{mg} / 24 \mathrm{~h}(\mathrm{~F})$. All data are expressed as mean $\pm \mathrm{SD}, * * * P<0.001, * * P<0.01$, vs. normal control, ${ }^{\circledR a} p<0.001$, ${ }^{\circledR a} P<0.01$, vs. alcohol treated, ${ }^{\# \# \#} P<0.001,{ }^{\# \#} P<0.01$, vs. disulfiram treated, ${ }^{\$ \$ \$} p<0.001, v s$. disulfiram + alcohol treated.

loss, contraction band necrosis, perivascular cuffing, increased intercalated space (widened intracellular spaces) and nuclei karyolysis in the ventricle photomicrographs. Instead of this, counter treatment of FBSLNP in the alcohol and disulfiram (either alone or in combination) pretreated animals showed significant protection in various structural abnormalities.
However, individually, FBSLNP did not show any effect in rat myocardium, as shown in Fig. 5(B-1 to B-8).

Indeed, alcohol and disulfiram consumption either alone or in combination lead to glomerulus structural damages including vacuolation of endothelial lining glomerular tufts, necrosis of epithelial lining renal tubules, atrophy of the glomerular tuft, hypertrophy of the glomerular tuft, and 


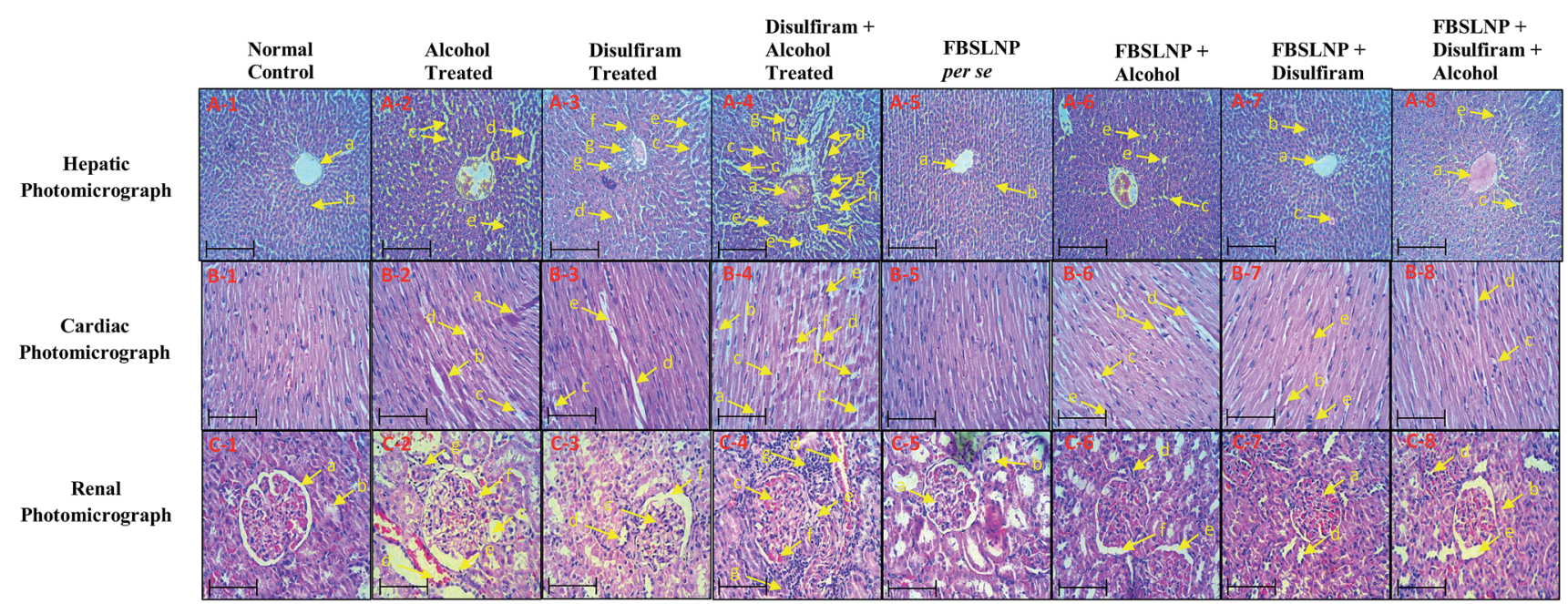

Fig. 5 Hepatic histological section (A-1 to A-8) with different treatment from each group highlights by arrow as: central vein ' $a$ ', hepatic cords 'b', swelling of hepatocytes ' $c$ ', Kupffer cell activation ' $d$ ', karyomegaly ' $e$ ', dysplasia of bile duct ' $f$ ', portal infiltration with inflammatory cell ' $g$ ' and hyperplasia and hyperactivation of epithelial lining bile duct ' $h$ '. B-1 to B-8 represents the histological section of cardiac tissues and arrow highlights: thrombus formation ' $a$ ', contraction band necrosis ' $b$ ', perivascular cuffing ' $c$ ', increased intercalated space (widened intracellular spaces) ' $d$ ' and nuclei karyolysis ' $e$ ', marked in different photomicrographs of different animal groups. Moreover, $C-1$ to $C-8$ represent the renal histological section and arrow highlights: normal glomerulus ' $a$ ', normal renal tubules ' $b$ ', vacuolation of endothelial lining glomerular tufts ' $c$ ', necrosis of epithelial lining renal tubules ' $d$ ', atrophy of glomerular tuft 'e', hypertrophy of glomerular tuft ' $f$ ', and congestion of renal blood vessels ' $g$ '. The histological examination was performed using Motic Microscope BA310 (Motic, USA) at 40× (scale bar $=100 \mu \mathrm{m})$.

congestion of renal blood vessels, compared to the kidney of normal control animals. Conversely, FBSLNP treated animals showed significant protection against glomerulus structural damages, as shown in Fig. 5(C-1 to C-8).

\section{Discussion}

Nanotechnology is an innovative approach in drug formulation, particularly in drug delivery. Certainly, several pathological conditions have been investigated by employing these new drug formulations (nanoparticles) having potent therapeutic action, targeted drug delivery and low toxicity. ${ }^{17}$ Moreover, SLNP have fascinating ability to improve plasma stability, biodistribution and subsequent bioavailability of drugs entrapped by altering the surface characteristics of nanoparticles. ${ }^{18}$ In addition, SLNP have exceptional admissibility, with nontoxic and biocompatible nature. SLNP are the colloidal drug carrier system equivalent to nanoemulsions, where liquid lipid is replaced by solid lipid at room temperature. ${ }^{18}$ Indeed, liver has RES, and hence formation of SLNP of $F$. benjamina was a suitable approach for targeted delivery. ${ }^{12,19}$ However, high dose of $F$. benjamina has been reported to cause sub-acute toxicity in a prolonged dosage regimen, and thus a need of an alternative strategy should be considered for desired therapeutic effect. FBSLNP could be a choice for better drug formulation, having higher potency, stability, controlled release and targeted drug delivery to inflamed tissue in comparison to the conventional dosage form. ${ }^{20}$

Evaluation of the physicochemical characteristics of a preformulation of $F$. benjamina extract explored the melting point, solubility and optimum partition coefficient in octanol/water. Product compatibility was further marked by the absence in drug/excipient interaction after storage for 3 months at $25^{\circ} \mathrm{C} \pm$ $2{ }^{\circ} \mathrm{C}, 60 \% \mathrm{RH} \pm 5 \%$. On the basis of varying ingredients concentration several formulation was composed (eight formulations including: FA, FB, FC, FD, FE, FF, FG and FG) and evaluated for entrapment efficiency, particle size, zeta potential and cumulative drug release (\% CDR). Entrapment efficiency is the ratio of the weight of drug entrapped into a carrier system to the total drug added, and it is important to achieve optimal entrapment. $^{21}$ Moreover, it has been revealed that the morphology of the nanoparticles should be spherical, which would ensure uniformity and less probability of dose dumping. For better bioavailability and therapeutic action, the particle size of the nanoparticles should be less than $100 \mathrm{~nm}$, which would allow easy permeation into body tissues and may produce positive clinical outcomes. Conversely, larger particles may not be engulfed by phagocytes and can cause difficulty in clearance. ${ }^{18,22}$ Moreover, as the pore size of liver fenestrate is about $100 \mathrm{~nm}$, nanoparticles having sizes less than this would be predominantly uptaken by the liver. ${ }^{23,24}$ Thus, particle size is a crucial index for size stability in the colloidal dosage form upon storage. Indeed, nanoparticles have larger surface area that facilitate the greater drug release and better bio-distribution. ${ }^{12,18}$ In addition, determination of the zeta potential is another essential factor for determining the physical stability of SLNP for liver targeting. ${ }^{22}$ Negatively charged nanoparticles are responsible for electronic stabilization and have more chances of intracellular accumulation in order to potentially target the liver tissue. ${ }^{23}$ The observed zeta potential of our prepared nanoformulation was found in the optimal range that can sustain the physical stability of nanoparticles due to the electric repulsion among particles. \% CDR is the critical factor for determining drug release in in vivo systems during a $12 \mathrm{~h}$ period. On the 
basis of results obtained in entrapment efficiency (68-93), particle size (range of 44 to $403 \mathrm{~nm}$ ), zeta potential $(-25.6$ to $-39.4 \mathrm{mV}$ ) and \% CDR of various formulations (76\% to $89 \%$ ) of FBSLNP, formulation FE (maximum entrapment efficiency, minimum particle size, suitable zeta potential and good \% CDR) was selected for further in vivo study. Furthermore, highdefinition microscopy based on a focused beam of electrons was carried out for a selected formulation (FE) of FBSLNP and revealed spherically shaped particles with sizes ranging from 20 to $50 \mathrm{~nm}$ due to the non-polar nature of stearic acid, which possessed a more stable morphology. ${ }^{24}$

As a matter of fact, hepatic injury is the most common pathological stage due to the prime site of metabolism and excretion. Chronic exposure to several drugs or junk food could result in liver fibrosis and cirrhosis. ${ }^{25}$ Alcohol is a psychoactive substance, which can result in addiction, and certainly causes toxicity in various organs including the liver, heart and renal tissues. ${ }^{26-28}$ Several clinical and preclinical reports revealed the associated altered biochemical parameters including increased level of SGOT, SGPT, ALP, serum albumin and total protein on chronic alcohol consumption. ${ }^{26,28}$ Similar biochemical alterations were measured in the present study, which confirm alcohol-induced hepatotoxicity. Disulfiram is a potent irreversible aldehyde dehydrogenase inhibitor, which is clinically known as antabuse across the world. ${ }^{29,30}$ The blockages of aldehyde dehydrogenase enzyme cause significant rise in aldehyde concentration, which gets accumulated in liver and leads to dual hepatic damage. ${ }^{30}$ This was confirmed by measuring the accumulated aldehyde concentration in disulfiram treated animal liver. Instead of various other chronic adverse drug reactions, aldehyde accumulation in the liver limits its clinical applications. Individual and combined administration of alcohol and disulfiram strongly perturbed various biochemical parameters including increased level of SGOT, SGPT, ALP, serum albumin, total protein, CKMB, LDH, cTnI, BUN, serum creatinine and microproteinuria, which significantly endorse the major damage in the liver and cardio-renal system. Moreover, accumulated aldehyde in liver confirmed the associated liver damage. Indeed, increased oxidative stress is another key factor for various organs being damaged. ${ }^{31}$ Individual and combined administration of alcohol and disulfiram resulted in significant elevation in oxidative stress and associated tissue damage.

The therapeutic potential of herbal drug nanoparticles against alcohol/disulfiram-induced liver toxicity remains an area for extensive scientific research. The current preclinical study provides substantial evidence of significant hepatoprotective potential of FBSLNP measured by restoration of altered biochemical parameters. SGOT, SGPT and ALP enzymes are highly sensitive serum biomarkers which directly represent the extent of hepatic damage. ${ }^{32}$ Moreover, FBSLNP significantly detoxify the accumulated aldehyde from liver measured by GCHS. Similarly, serum albumin and total protein levels were significantly increased by the treatment of FBSLNP. Substantial diminution of hepatic MDA and increased level of reduced GSH confirmed the anti-oxidant potential of FBSLNP. Hence detoxification of accumulated aldehyde from the liver and the anti-

oxidant potential of FBSLNP may be consider to be the possible mechanism of liver protection against alcohol/ disulfiram-induced hepatotoxicity. Histological outcomes further confirmed the structural restoration of liver tissue by FBSLNP treatment, as compared to alcohol/disulfiram treated animals.

High intake of alcohol with or without disulfiram treatment causes cardio-renal injury due to depletion of ATP and electron leakage from the respiratory chain. ${ }^{5}$ These free electrons combine with molecular oxygen and generate ROS. ${ }^{31}$ ROS can oxidize protein, DNA and membrane lipids and lead to cardiorenal damage. ROS mediated disruption may increase the release of CKMB, LDH and cTnI enzyme into the serum. ${ }^{\mathbf{1 6 , 3 1 , 3 3}}$ Furthermore, increased ROS concomitantly decreases the work functionality of renal system. ${ }^{\mathbf{1 6 , 3 3}}$ Altered glomerular filtration rate or deterioration in renal function was reflected by the increase in BUN and creatinine, as well as the occurrence of microproteinuria. ${ }^{16,33}$ The alcohol/disulfiram-induced deleterious effects on the renal system were indicated by the increased level of serum BUN, creatinine and the occurrence of microproteinuria. Cardio-renal protection was evident from the improved structural and functional parameters of the heart and kidney tissues. Aberrant cardio-renal biomarker levels were significantly restored by FBSLNP treatment in alcohol/ disulfiram treated animals.

In conclusion, the present study showed that FBSLNP is an appropriate formulation for targeted drug delivery to the liver, as revealed by entrapment efficiency, particle size, surface charge and in vitro release. $F$. benjamina has significant ability to detoxify the accumulated aldehyde in the liver, which is accumulated due to chronic alcoholism and disulfiram treatment. FBSLNP restored the structural and functional parameters of hepatic and cardio-renal tissues that were distorted by alcohol/ disulfiram administration. This constructive effect of FBSLNP to ameliorate alcohol/disulfiram-induced hepatic and cardiorenal toxicity may be due to their potential to detoxifying the accumulated aldehyde in the liver as well as their significant anti-oxidant, anti-inflammatory and anti-apoptotic effects.

\section{Conflict of interest}

The authors declare that there is no conflict of interest.

\section{Abbreviation}

FBSLNP

$\mathrm{ADH}$

SGOT

SGPT

GC-HS

CKMB

$\mathrm{LDH}$

cTnI

BUN

HDL

LDL
Ficus benjamina solid lipid nanoparticle Alcohol dehydrogenase

Serum glutamic oxaloacetic transaminase Serum glutamic pyruvic transaminase Headspace gas chromatograph

Creatine kinase MB

Lactate dehydrogenase

Cardiac troponin I

Blood urea nitrogen

High density lipoprotein

Low density lipoprotein 
MDA

TBARS

Malondialdehyde

GSH

ANOVA

TEM

\section{Acknowledgements}

The authors are grateful to the authority of the Amity Institute of Pharmacy, Amity University, Sector 125, Noida, India, for providing necessary facilities for the present study.

\section{References}

1 WHO, fact sheet 2015, http://www.who.int/mediacentre/ factsheets/fs349/en/, accessed April 18, 2017.

2 A. Sachdeva, M. Choudhary and M. Chandra, J. Clin. Diagn. Res., 2015, 9, 01-07.

$3 \mathrm{~W}$. Cheungpasitporn, C. Thongprayoon, W. Kittanamongkolchai, B. A. Brabec, O. A. O'Corragain, P. J. Edmonds and S. B. Erickson, QJM, 2015, 108, 539-548.

4 B. Maisch, Herz/Kreislauf, 2016, 41, 484-493.

5 A. I. Cederbaum, Clin. Liver Dis., 2012, 16, 667-685.

6 M. Setshedi, J. R. Wands and S. M. Monte, Oxid. Med. Cell. Longevity, 2010, 3, 178-185.

7 M. Imran, N. Rasool, K. Rizwan, M. Zubair, M. Riaz, M. ZiaUl-Haq, U. A. Rana, A. Nafady and H. Z. Jaafar, Chem. Cent. J., 2014, 8, 12.

8 N. Sirisha, M. Sreenivasulu, K. Sangeeta and C. M. Chetty, Int. J. PharmTech Res., 2010, 4, 2174-2182.

9 H. A. Almahy, M. Rabmanj, M. A. Sukari and A. M. Be Ali, Pertanika J. Sci. Technol., 2003, 11, 73-81.

10 V. K. Kanaujia, R. Irchhaiya, H. K. Singh, D. Kailasiya, M. Verma, R. D. Yadav and D. Shivhare, J. Nat. Prod. Plant Resour., 2011, 1, 59-69.

11 S. Hasti, E. Mora, R. Utami and L. U. Yulis, Procedia Chem., 2014, 13, 204-208.

12 S. Mukherjee, S. Ray and R. S. Thakur, Indian J. Pharm. Sci., 2009, 71, 349-358.

13 A. A. Nanji, K. Jokelainen, G. L. Tipoe, A. Rahemtulla, P. Thomas and A. J. Dannenberg, Am. J. Physiol.: Gastrointest. Liver Physiol., 2003, 284, 321-327.
14 L. Tampier, M. E. Quintanilla and Y. Israel, Alcohol.: Clin. Exp. Res., 2008, 32, 937-941.

15 C. Mayuren, V. V. Reddy, S. V. Priya and V. A. Devi, N. Am. J. Med. Sci., 2010, 2, 491-495.

16 A. K. Sharma, D. Khanna and P. Balakumar, Int. J. Cardiol., 2014, 172, 530-532.

17 W. H. De Jong and P. J. Borm, Int. J. Nanomed., 2008, 3, 133149.

18 M. Uner and G. Yener, Int. J. Nanomed., 2007, 2, 289-300.

19 A. Ali Khan, J. Mudassir, N. Mohtar and Y. Darwis, Int. J. Nanomed., 2013, 8, 2733-2744.

20 S. Hua, Front. Pharmacol., 2014, 5, 138.

21 T. B. Dudala, P. R. Yalavarthi, H. C. Vadlamudi, J. Thanniru, G. Yaga, N. L. Mudumala and V. K. Pasupati, Int. J. Pharm. Invest., 2014, 4, 149-155.

22 M. Lundqvist, J. Stigler, G. Elia, I. Lynch, T. Cedervall and K. A. Dawson, Proc. Natl. Acad. Sci. U. S. A., 2008, 105, 14265-14270.

23 D. Peer, Nanotechnology for the Delivery of Therapeutic Nucleic Acids, volume 4 of Pan Stanford Series on Biomedical Nanotechnology, CRC Press, 2013, ISBN 9814411051, 9789814411059.

24 S. Barua and S. Mitragotri, Nano Today, 2014, 9, 223-243.

25 S. Manzo-Avalos and A. Saavedra-Molina, Int. J. Environ. Res. Public Health, 2010, 7, 4281-4304.

26 A. U. Tatiya, S. J. Surana, M. P. Sutar and N. H. Gamit, Pharmacogn. Res., 2012, 4, 50-56.

27 S. Y. Kim and A. Moon, Biomol. Ther., 2012, 20, 268-272.

28 V. P. Singh, N. Singh and A. S. Jaggi, Korean J. Physiol. Pharmacol., 2013, 17, 347-357.

29 P. Marcato, C. A. Dean, C. A. Giacomantonio and P. W. Lee, Cell Cycle, 2011, 10, 1378-1384.

30 V. Koppaka, D. C. Thompson, Y. Chen, M. Ellermann, K. C. Nicolaou, R. O. Juvonen, D. Petersen, R. A. Deitrich, T. D. Hurley and V. Vasiliou, Pharmacol. Rev., 2012, 64, 520-539.

31 A. K. Sharma, G. Taneja, D. Khanna and S. K. Rajput, $R S C$ Adv., 2015, 5, 57267-57276.

32 S. Gowda, P. B. Desai, V. V. Hull, A. A. Math, S. N. Vernekar and S. S. Kulkarni, Pan Afr. Med. J., 2009, 3, 17.

33 A. K. Sharma, A. Kumar, G. Taneja, U. Nagaich, A. Deep and S. K. Rajput, RSC Adv., 2016, 6, 36870-36880. 